\title{
Clinical utility of naloxegol in the treatment of opioid-induced constipation
}

\author{
This article was published in the following Dove Press journal: \\ Journal of Pain Research \\ 12 June 2015 \\ Number of times this article has been viewed
}

\author{
Heather C Bruner' \\ Rabia S Atayee ${ }^{2}$ \\ Kyle P Edmonds ${ }^{3}$ \\ Gary T Buckholz ${ }^{3}$
}

'Scripps Health and University of California San Diego, Joint Hospice and Palliative Medicine Fellowship, San Diego, CA, USA; ${ }^{2}$ University of California San Diego, Skaggs School of Pharmacy and Pharmaceutical Sciences, La Jolla, CA, USA;

${ }^{3}$ Department of Medicine, University of California San Diego, Doris A Howell Palliative Care Service, La Jolla, CA, USA
Correspondence: Rabia S Atayee University of California San Diego, Skaggs School of Pharmacy and Pharmaceutical Sciences, 9500 Gilman Drive, MC 0675, La Jolla, CA 92093-0675, USA

$\mathrm{Tel}+$ I 8588223549

Fax +l 8588225624

Email ratayee@ucsd.edu
Abstract: Opioids are a class of medications frequently used for the treatment of acute and chronic pain, exerting their desired effects at central opioid receptors. Agonism at peripherally located opioid receptors, however, leads to opioid-induced constipation (OIC), one of the most frequent and debilitating side effects of prolonged opioid use. Insufficient relief of OIC with lifestyle modification and traditional laxative treatments may lead to decreased compliance with opioid regimens and undertreated pain. Peripherally acting mu-opioid receptor antagonists (PAMORAs) offer the reversal of OIC without loss of central pain relief. Until recently, PAMORAs were restricted to subcutaneous route or to narrow patient populations. Naloxegol is the first orally dosed PAMORA indicated for the treatment of OIC in noncancer patients. Studies have suggested its efficacy in patients failing traditional constipation treatments; however, insufficient evidence exists to establish its role in primary prevention of OIC at this time.

Keywords: opioid-induced bowel dysfunction, chronic pain, peripherally-acting mu-opioid antagonist, bowel care, OIC, OIBD

\section{Introduction}

Opioids, a mainstay of treatment for acute and chronic pain, are the most commonly prescribed class of drugs in the United States, with more than 240 million prescriptions per year. ${ }^{1}$ Opioids exert their desired analgesic effects via mu-receptors in the central nervous system (CNS). Opioid stimulation of mu-receptors found throughout the gastrointestinal (GI) tract, however, may result in the constellation of adverse effects known as opioidinduced bowel dysfunction (OIBD). Enteric opioid stimulation results in decreased propulsive muscular contraction, inhibition of water and electrolyte secretions, and increased rectal sphincter tone with decreased rectal contractility. ${ }^{2}$ OIBD is manifested by a wide range of symptoms such as dry mouth, gastroesophageal reflux, vomiting, bloating, abdominal pain, anorexia, hard stools, constipation, and incomplete evacuation. ${ }^{3}$ Unfortunately, tolerance to GI side effects does not typically develop and several studies have confirmed a high prevalence of OIBD in patients taking opioid pain medication. ${ }^{3,4}$

The most common manifestation of OIBD is opioid-induced constipation (OIC). ${ }^{1}$ Estimations of its prevalence vary widely, but systematic reviews suggest ranges between $60 \%$ and $90 \%$ of subjects with cancer-related opioid use and between $40 \%$ and $60 \%$ of patients taking opioids for nonmalignant pain. ${ }^{1} \mathrm{OIC}$ is also one of the most debilitating side effects of opioid therapy. ${ }^{1}$ OIC has been shown to impair patients' ability to carry out their activities of daily living. ${ }^{5-7}$ It leads to increased missed work and diminished work productivity, as well as lower levels of overall health, healthrelated quality of life (QoL), and well-being. ${ }^{4-7}$ Patients taking opioids for more than 
6 months who suffered from OIC were more likely to take time off work or feel impaired in their work and domestic performance than those who did not develop OIC. ${ }^{4}$ OIC led to hospitalization in $16 \%$ of cancer patients and pain in $75 \%$ of patients with subsequent changes in laxative and opioid regimens. ${ }^{6}$ One estimation has it that $30 \%$ of patients reduce or stop taking opioids as a result of OIC. ${ }^{8}$

OIC has been traditionally treated identically to functional constipation with a combination of lifestyle modification and medications. Lifestyle modifications can include increased fiber intake, increased liquid intake, as well as physical activity. ${ }^{4,9}$ Paradoxically, fiber intake may worsen OIC as it adds bulk to stool volume. ${ }^{4}$ Because nonpharmacologic measures do not typically adequately control OIC, laxatives, such as stimulants and stool softeners, are the treatments of choice. Stimulants include senna or bisacodyl, which increase smooth muscle contraction. Stool softeners include surfactants such as docusate, lubricants such as mineral oil and osmotics, such as lactulose or polyethylene glycol (PEG). ${ }^{4}$ Softeners may be used in combination with stimulants for prevention or treatment of OIC, but alone may be ineffective. ${ }^{4}$ Although typical practice is to prescribe laxatives concurrently with opioids, studies suggest that $54 \%$ of patients on both medications fail to symptomatically improve greater than half the time. ${ }^{4}$ Furthermore, a 2011 Cochrane Collaboration review failed to demonstrate the superiority of one laxative over any other. ${ }^{10}$ Opioid reversal agents, such as naloxone, have been typically used parenterally to achieve rapid, central opioid antagonism to treat life-threatening opioid overdoses. Meissner et al suggested in a 2009 randomized, placebo-controlled trial that a sustained-release, oral preparation of naloxone coadministered with long-acting oxycodone was more effective in the treatment and prevention of OIC than placebo without any reduction in analgesia. ${ }^{11}$

A newer class of agents, seeking to address the underlying pathophysiology of OIBD and OIC, are called peripherally acting mu-opioid receptor antagonists (PAMORAs). The advantage of medications in this class is their lack of CNS penetration. Thus PAMORAs preserve centrally mediated opioid analgesic effects while reversing or preventing the unwanted peripheral side effect of OIC. This class includes commercially available methylnaltrexone and alvimopan. Methylnaltrexone has been shown to confer benefit over placebo in inducing bowel movements (BMs) in patients taking opioids who have failed traditional constipation treatment. ${ }^{10,12}$ Its utility, however, is limited by its subcutaneous administration route and its indication for use in advanced illness. ${ }^{3}$ Additionally, reports of GI perforation related to methylnaltrexone use have concerned some clinicians. A 2010 letter to the editors of Journal of Pain and Symptom Management reported seven cases of bowel perforation temporally related to administration of methylnaltrexone. Several of these patients, however, had documented GI abnormalities such as peptic ulcer, volvulus, and underlying colon cancer, making causal relationship to methylnaltrexone difficult to prove. ${ }^{13}$ Alvimopan is only approved in the United States for the treatment of postoperative ileus. ${ }^{3}$ Its use is also limited due to the potential for myocardial infarction and required FDA risk evaluation and mitigation strategy (REMS) program. ${ }^{14}$ Naloxegol, the focus of this review, is a new oral, once-daily PAMORA.

\section{Pharmacology of naloxegol}

Naloxegol is a PEGylated derivative of naloxone that functions as a PAMORA. ${ }^{15,16}$ When administered at the recommended dosing, naloxegol antagonizes the mu-receptor in tissues such as the GI tract, thereby decreasing the constipating effects of opioids. In vitro studies have demonstrated low affinity for the delta- or kappa-opioid receptors. ${ }^{1}$ The presence of the PEG moiety in naloxegol reduces its passive permeability as compared with naloxone. Naloxegol is also a substrate for the P-glycoprotein transporter. Due to the reduced permeability and increased efflux of naloxegol across the blood-brain barrier, related to $\mathrm{P}$-glycoprotein transporter, the $\mathrm{CNS}$ penetration of naloxegol is negligible and in the GI tract it reduces OIC without reversal of central analgesic effect. ${ }^{15,16}$

\section{Pharmacokinetic summary}

The recommended dose of naloxegol is $25 \mathrm{mg}$ orally oncedaily. ${ }^{16,17}$ Following oral administration, naloxegol exhibits a first peak in concentration at less than 2 hours. Interestingly, due to enterohepatic recycling, a second peak occurs in most patients $0.4-3$ hours after the first peak. ${ }^{16}$ Both maximum plasma concentration $\left(C_{\max }\right)$ and area under the plasma concentration curve (AUC) of naloxegol increase with high-fat meals; hence, administration guidelines recommend dosing 1 hour before meals (empty stomach). Grapefruit juice can also increase naloxegol plasma concentration and should be avoided during naloxegol treatment. ${ }^{16}$

Metabolism of naloxegol primarily occurs at the CYP3A4 enzyme resulting in metabolites whose effects are yet unknown. Naloxegol is contraindicated with concomitant use of strong CYP3A4 inhibitors and inducers. ${ }^{18}$ Dose adjustment of $12.5 \mathrm{mg}$ administered orally is recommended in moderate CYP3A4 inhibitors while no dose adjustment is recommended with weak CYP3A4 inhibitors. ${ }^{16}$ 
Naloxegol is primarily excreted in the feces and its half-life extends from 6 to 11 hours. ${ }^{16}$ Patients with mildto-moderate hepatic impairment (as defined by Child-Pugh scores of A and B) showed slight decreases in AUC compared to those with normal hepatic function. ${ }^{19}$ Naloxegol's product information does not recommend any dose adjustments in mild-to-moderate hepatic impairment. The medication has not been studied in severe hepatic impairment and thus product information does not recommend its use in these patients. ${ }^{16}$

Patients with moderate-to-severe renal impairment had increased AUCs and those with severe renal impairment had increased $\mathrm{C}_{\text {max }}$ and decreased clearance. ${ }^{20}$ Renal elimination is a minor route of naloxegol and there was no correlation between renal clearance and total clearance. Naloxegol was not effectively removed during standard 4-hour hemodialysis. ${ }^{20}$ Dosing recommendations for creatinine clearance $<60 \mathrm{~mL} / \mathrm{min}$ are to start with $12.5 \mathrm{mg}$ daily and, if tolerated and symptoms of OIC persist, to increase to $25 \mathrm{mg}$ daily. ${ }^{20}$

\section{Efficacy studies}

The development of naloxegol has included two Phase I, one Phase II, and two Phase III trials to elucidate the pharmacokinetics, clinical efficacy, and safety of this PAMORA. There was an additional Phase III extension trial to assess for long-term safety and effectiveness. Key trials are summarized in Table 1.

Two, identical, multicenter, double-blind, placebocontrolled, Phase III studies (KODIAC-04 and KODIAC-05) evaluated the 12-week response rate of $12.5 \mathrm{mg}, 25 \mathrm{mg}$, and placebo. ${ }^{2}$ Eligible patients included those with confirmed OIC who did not have uncontrolled pain, cancer in previous 5 years, use of other constipation-promoting medications, evidence of bowel obstruction, or a baseline elevated risk of bowel perforation. ${ }^{2}$ For these studies, the authors defined OIC as $<3$ spontaneous BMs ( $\mathrm{sBMs}$ ) in a week accompanied by hard stools, difficult passage of stool, sensation of incomplete evacuation, or anorectal obstruction for $25 \%$ of all BMs in the 4 weeks prior to screening. ${ }^{2}$ The authors defined a response as $\geq 3 \mathrm{sBMs}$ in a week accompanied by an increase in sBMs over baseline by at least one; these needed to occur in 9 out of 12 weeks of the trial as well as in 3 out of 4 of the final weeks of the trial. This primary endpoint was significantly achieved for both doses in the KODIAC-04 trial and for the $25 \mathrm{mg}$ dose in the KODIAC-05 trial.

There were multiple efficacy-related secondary endpoints, including time to sBM following first dose, mean number of sBMs per week as well as mean number of days per week with $\geq 1$ sBM, severity of straining, hardness of stool, and need for rescue laxative use. ${ }^{2}$ All these endpoints demonstrated favorable response at the $25 \mathrm{mg}$ dose, though the results were more mixed in the $12.5 \mathrm{mg}$ dose group. At the $25 \mathrm{mg}$ dose, time to first sBM following medication administration was 5.9 hours in KODIAC-04 and 12 hours in KODIAC-05 as compared to nearly 40 hours in the placebo group. ${ }^{2}$

Additionally, Chey et al analyzed a subgroup of patients with prior inadequate response to laxatives. They defined this group as those who took a laxative of any class for $\geq 4$ days out of the 2 weeks prior to start of study who still had moderate, severe, or very severe OIBD symptoms on the intake questionnaire. ${ }^{2}$ This population had a significant response rate at both doses of naloxegol in KODIAC-04, with the $25 \mathrm{mg}$ group having a 19.9\% improved response over placebo. Again, KODIAC-05 demonstrated response in the $25 \mathrm{mg}$ group, but not in the $12.5 \mathrm{mg}$ group. ${ }^{2}$

\section{Safety and tolerability}

Adverse events with naloxegol have proven to be overall more common at the $25 \mathrm{mg}$ dose and primarily include GI complaints such as abdominal pain, diarrhea, nausea, and vomiting. ${ }^{2,21}$ These unwanted effects were overall mild to moderate in severity and typically occurred soon after the first dose of naloxegol. ${ }^{2}$ At the $25 \mathrm{mg}$ dose in both KODIAC-04 and KODIAC-05, approximately $10 \%$ of those participants experiencing adverse events discontinued naloxegol use. Diarrhea was the most common reason to discontinue in KODIAC-04 while abdominal pain lead to the most discontinuation in KODIAC-05, though there is no comment on where in the course of the trial these patients stopped taking naloxegol. ${ }^{2}$ There was no change in overall pain score or mean daily opioid doses. Potential symptoms of drug withdrawal were rare and were felt to be artifacts of the scoring system that were primarily driven by GI symptoms. ${ }^{2}$

An additional multicenter, open-label, randomized, parallel-group, Phase III study monitored patients on naloxegol over a 52-week period (Table 1). This study demonstrated, again, an approximately $10 \%$ discontinuation rate of the study drug as a result of diarrhea, abdominal pain, and vomiting. ${ }^{21}$ The majority of participants experienced some naloxegolrelated unwanted event, though most were considered mild to moderate in intensity. ${ }^{21}$ They found no naloxegol-related change in pain scores, mean opioid daily dose, or opioid withdrawal scores. ${ }^{21}$

In both studies, there were no adjudicated major events that were found to be linked to naloxegol nor were there instances of actual or potential bowel perforation. ${ }^{2,21}$ However, 


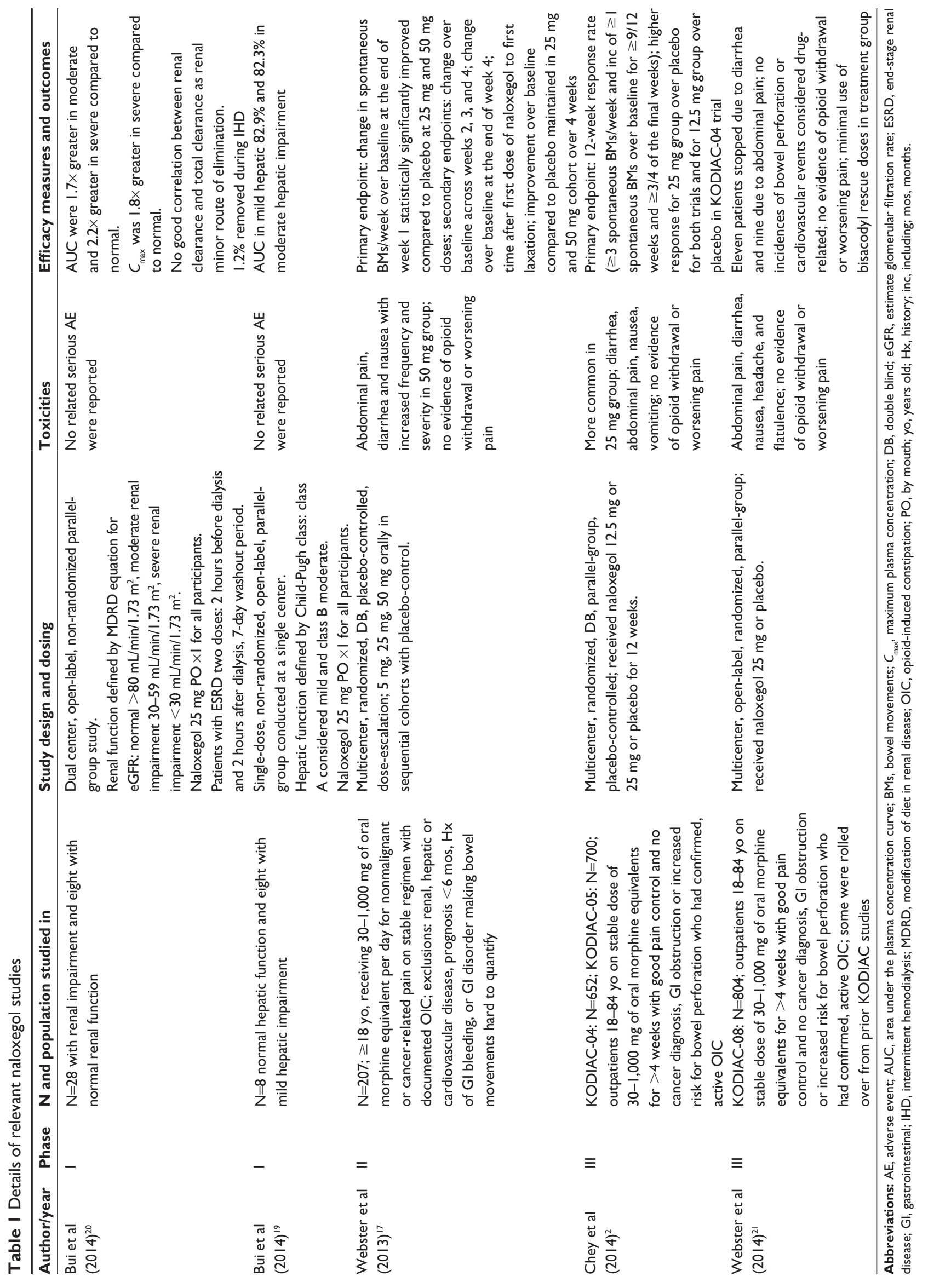


as mentioned previously, patients with bowel obstruction or gastrointestinal disease that could put them at risk for compromised bowel wall integrity were excluded from the study. $^{2}$

Finally, an additional, randomized, positive- and placebocontrolled crossover study with 52 healthy men receiving doses as high as $150 \mathrm{mg}$ of naloxegol demonstrated no QT/ QTc interval increase $>30 \mathrm{~ms}^{22}$

\section{Patient-focused perspectives}

Clinical trials of naloxegol that address patient-focused outcomes are limited. Webster et al conducted a Phase II, double-blind, randomized, placebo-controlled dose-escalation study of naloxegol that included patient-oriented outcomes assessments. ${ }^{17}$ In addition to assessment for the primary endpoints, patients also completed several subjective questionnaires: the Patient Assessment of ConstipationSymptom (PAC-SYM) questionnaire, which assesses abdominal, rectal, and stool symptoms; the Patient Assessment of Constipation Quality-of-Life (PAC-QoL) questionnaire, which encompasses physical discomfort, worries/concerns, psychosocial discomfort, and satisfaction, and the ShortForm Health Survey (SF-36), which yields an 8-scale profile of functional health and well-being scores.

Webster et al found statistically significant lower mean PAC-SYM scores for rectal symptoms in both the $25 \mathrm{mg}$ and $50 \mathrm{mg}$ groups. ${ }^{17}$ Patients in the $25 \mathrm{mg}$ cohort experienced statistically significantly lower PAC-QoL mean scores for physical discomfort as well as statistically significant improvement in multiple items on the SF-36 survey, including improvement in physical functioning, mental health, social functioning, and vitality. ${ }^{17}$

It is reassuring that, across the studies, most participantreported adverse events were described as mild to moderate and transient in nature. , 17,21 There were no statistically significant changes in participant-reported pain, opioid usage, or withdrawal symptoms across any of the dosage groups., ${ }^{2,17,21}$

Subjective symptoms of straining with stools and stool hardness were secondary endpoints in the study by Chey et al; both symptoms of OIC improved most at the $25 \mathrm{mg}$ level, but were also found significantly improved over the placebo group in the $12.5 \mathrm{mg}$ group in KODIAC- $04 .^{2}$

Unfortunately, an additional trial (KODIAC-06) of naloxegol in patients with cancer-associated pain succeeded in recruiting only $4 \%$ of planned participants and was therefore discontinued early. ${ }^{23}$

Naloxegol was initially a schedule II controlled substance because it is structurally related to noroxymorphone.
Its manufacturers sent a petition to the US Drug Enforcement Administration to deschedule. As of January 23, 2015, naloxegol was descheduled and the prescribing information has been updated. ${ }^{24}$

\section{Conclusion and place in therapy}

Opioid medications are commonly prescribed for acute and chronic pain and have the unfortunate side effect of OIBD and OIC. ${ }^{1}$ Significant morbidity and negative impact on QoL are associated with OIC. ${ }^{4-7}$ PAMORAs are a drug class that show considerable promise in treating OIC, given the mechanism of preventing the underlying problem of peripheral mu-receptor agonism, which causes constipation as well as other OIBD symptoms. ${ }^{4}$ In contrast, traditional laxative therapies manage the subsequent effects of OIBD and OIC rather than directly focusing on preventing or reversing the underlying problem.

Naloxegol may have a clear advantage over other PAMORAs for several reasons. Its oral route makes it ideal for daily use in the outpatient setting with the goal of preventing OIC. In comparison, methylnaltrexone's subcutaneous route makes it impractical for daily use. Subsequently, methylnaltrexone is often thought of as a rescue medication when a patient taking opioid medication(s) has gone a number of days without a BM. While alvimopan is an oral alternative, it is currently only approved for postoperative ileus, has potential cardiac issues, and requires REMS. ${ }^{14}$ Naloxegol, on the other hand, has no apparent cardiac issues and avoided scheduled status, which will make prescribing easier. Though naloxegol will be more expensive to purchase than traditional laxative therapies, it remains unclear whether more effective prevention of OIBD and OIC can decrease the overall cost burden associated with this opioid side effect.

Many of naloxegol's potential side effects (eg, diarrhea, abdominal cramping) are likely related to the drug doing its intended job. It remains to be seen whether the increased $C_{\max }$ and AUC when taken with high-fat meals will have any significant clinical or adverse effects. Even though it is metabolized by the liver and excreted in the feces, dose changes are not recommended for mild-to-moderate hepatic impairment. For renal impairment, dose adjustment is recommended. This mirrors recommendations for most opioids making it more convenient for the prescriber. For example, when a provider considers dose adjustment of morphine (or rotation to another opioid) due to renal impairment, it will be logical to consider dose adjustment of naloxegol at the same time.

Given the known studies to date, it appears naloxegol has been proven to be very helpful for noncancer patients 
with constipation who have failed traditional laxatives. Additionally, it would be expected that morbidity associated with OIC decreases and QoL improves. However, it remains unclear if naloxegol is superior to traditional laxative regimens as first line therapy for OIC. Head-to-head trials of naloxegol vs traditional laxative regimens are needed to provide clarification on whether it should be considered first line therapy for OIC. The vonRoenn et al study attempted to evaluate naloxegol for OIC in cancer patients, but ended early due to low recruitment. ${ }^{23}$ Although naloxegol has not been extensively studied in cancer patients, it is likely but unproven that similar benefits would be observed for OIC in those patients. Hopefully, future studies will explore the potential role of this new agent in patients with cancer.

\section{Disclosure}

The authors report no conflicts of interest in this work.

\section{References}

1. Tack J, Corsetti M. Naloxegol for the treatment of opioid-induced constipation. Expert Rev Gastroenterol Hepatol. 2014;8(8):855-861.

2. Chey WD, Webster L, Sostek M, Lappalainen J, Barker PN, Tack J. Naloxegol for opioid-induced constipation in patients with noncancer pain. N Engl J Med. 2014;370(25):2387-2396.

3. Poulsen JL, Brock C, Olesen AE, Nilsson M, Drewes AM. Clinical potential of naloxegol in the management of opioid-induced bowel dysfunction. Clin Exp Gastroenterol. 2014;7:345-358.

4. Kumar L, Barker C, Emmanuel A. Opioid-induced constipation: pathophysiology, clinical consequences, and management. Gastroenterol Res Pract. 2014;2014:141737.

5. Bell T, Annunziata K, Leslie JB. Opioid-induced constipation negatively impacts pain management, productivity, and health-related quality of life: findings from the National Health and Wellness Survey. J Opioid Manag. 2009;5(3):137-144.

6. Abramowitz L, Beziaud N, Labreze L, et al. Prevalence and impact of constipation and bowel dysfunction induced by strong opioids: a crosssectional survey of 520 patients with cancer pain: DYONISOS study. J Med Econ. 2013;16(12):1423-1433.

7. Coyne KS, LoCasale RJ, Datto CJ, Sexton CC, Yeomans K, Tack J. Opioid-induced constipation in patients with chronic noncancer pain in the USA, Canada, Germany, and the UK: descriptive analysis of baseline patient-reported outcomes and retrospective chart review. Clinicoecon Outcomes Res. 2014;6:269-281.

8. Olesen AE, Drewes AM. Validated tools for evaluating opioid-induced bowel dysfunction. Adv Ther. 2011;28(4):279-294.

9. Tarumi Y, Wilson MP, Szafran O, Spooner GR. Randomized, doubleblind, placebo-controlled trial of oral docusate in the management of constipation in hospice patients. J Pain Symptom Manage. 2013;45(1): $2-13$.

Journal of Pain Research

\section{Publish your work in this journal}

The Journal of Pain Research is an international, peer-reviewed, open access, online journal that welcomes laboratory and clinical findings in the fields of pain research and the prevention and management of pain. Original research, reviews, symposium reports, hypothesis formation and commentaries are all considered for publication.

Submit your manuscript here: http://www.dovepress.com/journal-of-pain-research-journal
10. Candy B, Jones L, Goodman ML, Drake R, Tookman A. Laxatives or methylnaltrexone for the management of constipation in palliative care patients. Cochrane Database Syst Rev. 2011;1:CD003448.

11. Meissner W, Leyendecker P, Mueller-Lissner S, et al. A randomized controlled trial with prolonged-release oral oxycodone and naloxone to prevent and reverse opioid-induced constipation. Eur J Pain. 2009; 13:56-64.

12. Thomas J, Karver S, Cooney GA, et al. Methylnaltrexone for opioidinduced constipation in advanced illness. N Engl J Med. 2008;358(22): 2332-2343.

13. Mackey AC, Green L, Greene P, Avigan M. Methylnaltrexone and gastrointestinal perforation. J Pain Symptom Manage. 2010;40(1): e1-e3.

14. Entereg (alvimopan) [package insert]. Lexington, MA: Cubist Pharmaceuticals, Inc.; 2013.

15. Eldon MA, Song D, Neumann TA, et al. NKTR-118 (oral PEG-naloxol), a PEGylated derivative of naloxone: demonstration of selective peripheral opioid antagonism after oral administration in preclinical models. Poster presented at: 18th Annual Clinical Meeting of the American Academy of Pain Management; September 2007; Las Vegas, NV, USA.

16. Movantik (naloxegol) [package insert]. Wilmington, DE: AstraZeneca Pharmaceuticals LP; 2014.

17. Webster L, Dhar S, Eldon M, Masuoka L, Lappalainen J, Sostek M. A phase 2, double-blind, randomized, placebo-controlled, doseescalation study to evaluate the efficacy, safety, and tolerability of naloxegol in patients with opioid-induced constipation. Pain. 2013; 154(9):1542-1550.

18. Flockhart D. Drug Interactions: Cytochrome P450 Drug Interaction Table - P450 Drug Interaction Table: Abbreviated "Clinically Relevant” Table; 2007 [June 30, 2011; cited July 30, 2011]. Available from: http://medicine.iupui.edu/clinpharm/DDIs/ClinicalTable.aspx.

19. Bui K, She F, Sostek M. The effects of mild or moderate hepatic impairment on the pharmacokinetics, safety, and tolerability of naloxegol. J Clin Pharmacol. 2014;54(12):1368-1374.

20. Bui K, She F, Sostek M. The effects of renal impairment on the pharmacokinetics, safety, and tolerability of naloxegol. J Clin Pharmacol. 2014;54(12):1375-1382.

21. Webster L, Chey WD, Tack J, Lappalainen J, Diva U, Sostek M. Randomised clinical trial: the long-term safety and tolerability of naloxegol in patients with pain and opioid-induced constipation. Aliment Pharmacol Ther. 2014;40(7):771-779.

22. Gottfridsson C, Carlson G, Lappalainen J, Sostek M. Evaluation of the effect of naloxegol on cardiac repolarization: a randomized, placeboand positive-controlled crossover thorough QT/QTc study in healthy volunteers. Clin Ther. 2013;35(12):1876-1883.

23. vonRoenn JH, Tack J, Barker PN, Lowe ES, Fleischmann C, Sostek M. Challenges in patient recruitment during KODIAC-06, a randomized, placebo-controlled, double-blind, multicenter, phase 3 trial of naloxegol in patients with neoplasia and opioid-induced constipation (OIC). Poster presented at: MASCC/ISOO International Symposium on Supportive Care in Cancer; June 2013; Berlin, Germany.

24. http://www.deadiversion.usdoj.gov/fed_regs/rules/2015/fr0123_3.htm [web page on the internet]. Washington, DC: US Department of Justice Office of Diversion Control; 2014 [Filed October 28, 2014]. Available from: http://perma.cc/TD4L-CFKW. Accessed January 26, 2015.

The manuscript management system is completely online and includes a very quick and fair peer-review system, which is all easy to use. Visit http://www.dovepress.com/testimonials.php to read real quotes from published authors. 